\title{
Marsile Ficin et Hermès Trismégiste. Quelques notes sur la traduction du Pimandre dans la Vulgata de Ficin.
}

\author{
ILANA KLUTSTEIN
}

$\mathrm{L}$

_orsqu'on évoque Marsile Ficin traducteur, c'est principalement aux oeuvres de Platon que l'on pense. Bien qu'il ait bâti un système philosophique reposant sur la synthèse du néoplatonisme et de la foi chrétienne, son vrai titre de gloire est celui qui fait de lui un messager: grâce à ses traductions, le monde savant redécouvrira une philosophie grecque qui pendant des siècles s'était effacée devant celle d'Aristote. Elle prendra désormais une part de plus en plus importante dans l'histoire de la pensée.

Mais Ficin n'a pas traduit que Platon. Nous possédons de lui des traductions de Plotin, Proclus, Porphyre, Iamblique et autres néoplatoniciens, et également des textes qui pour lui sont des révélations d'anciens sages, précurseurs de l'enseignement platonicien. Il n'est besoin de citer que quelques phrases de son introduction au Pimandre, pour se convaincre de sa foi en l'existence d'une transmission de la vraie sagesse.

"[Mercurius Trismegistus] Primus igitur Theologiae appellatus est autor. eum secutus Orpheus, secundas antiquae Theologiae partes obtinuit. Orphei sacris initiatus est Aglaophemus. Aglaophemo successit in Theologia Pythagoras, quem Philolaus sectatus est, divi Platonis nostri praeceptor."1

Ces sages-philosophes n'ont pas tous la même importance aux yeux de Ficin. Certains sont souvent cités, textuellement ou non; d'autres ont droit à une traduction intégrale. C'est ainsi que nous pouvons lire tout le texte latin du Corpus Hermeticum, que Ficin nomme "Pimander" d'après le nom du premier traité.

Dans une précédente étude, ${ }^{2}$ nous avons minutieusement examiné les diverses citations d'Orphée et de Zoroastre contenues dans la Théologie platonicienne. Travaillant dans le cadre d'une comparaison avec d'autres traductions du même texte grec, nous avons pu arriver à des conclusions significatives sur la méthode de Ficin dans sa traduction: d'une façon suivie, 
il tronque souvent les phrases ou en choisit les fragments qui l'intéressent particulièrement dans le cours de son argumentation. D'autre part, le sens original des mots grecs est souvent changé, intentionnellement ou non, ou bien encore le concept latin utilisé dans le nouveau contexte entraîne des connotations différentes de celles de l'original grec.

L'objet de cet article est d'étudier la traduction de Ficin dans le Corpus Hermeticum. Ici, nous avons affaire à une situation différente: Ficin ne rapporte plus de citations isolées, c'est une traduction intégrale, à partir d'un manuscrit grec que nous savons avoir été entre ses mains: mieux, c'est une traduction exécutée à la demande de Cosme de Medicis, intéressé par ce nouveau manuscrit. La méthode de Ficin sera-t-elle affectée par le fait d'une traduction intégrale? C'est ce que nous voulons tenter de mettre en lumière.

Les faits entourant l'exécution de ce travail nous sont connus ${ }^{3}$ Ce manuscrit étant parvenu de Macédoine aux mains de Cosme, il demanda à Ficin de retarder sa traduction de Platon, pour entreprendre celle beaucoup plus courte d'Hermès. Ficin s'attela au travail avec l'enthousiasme d'un néophyte. Cette traduction était la première qu'il effectuait, et comme nous le verrons par la suite, ce point se révèlera être important. ${ }^{4}$

Nous savons que le manuscrit grec sur lequel il basait sa traduction, (Cod. Laur. 71,33) appartenait au type A décrit par Reitzenstein, ${ }^{5}$ mais tous les manuscrits suivent plus ou moins le même archétype. Nous nous proposons toutefois de collationner dans un proche avenir le manuscrit grec, pour relever les différences qui pourraient être signficatives.

En ce qui concerne le texte latin lui-même, nous suivons l'édition des Opera Omnia faite à Bâle, en 1576. Cette édition est relativement tardive et comporte beaucoup d'erreurs dues aux copistes ou aux imprimeurs. Pour avoir une idée de l'infidélité de cette version par rapport à la traduction originale de Ficin, il faut se reporter à la note de $\mathrm{M}$. Purnell, ${ }^{6}$ où celui-ci montre de façon convaincante que le texte de Ficin a été modifié, probablement mal recopié à certains endroits. ${ }^{7}$ La traduction italienne de Tommaso Benci fidele reproduction du latin, reconstitue, quand il y a doute, les termes originaux de Ficin. Suivant ces directives, nous avons effectivement retrouvé des erreurs dues aux copistes, mais qui peuvent avoir parfois une influence tout à fait innattendue sur la réception du texte. ${ }^{8}$ Une étude de cette traduction ne peut donc être complète si elle ne se base sur les manuscrits et sur les premières éditions de Trévise et Ferrare, plus exactes que celle de Bâle, que l'on peut considérer comme un "Vulgata". Ceci n'est donc qu'un premier travail que nous comptons approfondir dans un stade ultérieur. 
Les remarques qui vont suivre ne concernent que le premier traité du Pimandre-un des plus longs et des plus importants-où figure toute une cosmogonie et encore bien d'autres éléments philosophiques. Ce ne sont que des notes préliminaires, mais il semble bien que nous retrouvions une tendance ficinienne connue, analogue à celle que nous avons découverte dans les textes précédemment étudiés.

Il nous faut cependant garder à l'esprit ce que décrit si bien F. Yates ${ }^{9}$ lorsqu'elle analyse le Pimandre de Ficin. Nous savons, nous, modernes, que ces textes païens sont apocryphes, mais pour comprendre l'impact réel qu'ils ont eu sur les érudits de la Renaissance et sur Ficin en particulier, n'oublions pas que ces derniers croyaient en leur authenticité, ce qui influençait la compréhension qu'ils en avaient. Dans certains passages du Pimandre, le rapprochement avec des thèmes bibliques et chrétiens est parfois si saisissant que l'on peut imaginer sans effort l'enthousiasme de Ficin à les traduire. Pouvait-il rêver meilleure preuve de l'évidence de sa foi?

Avant d'aborder l'étude de la traduction elle-même, il nous faut d'abord éliminer les erreurs dues au copiste. Le paragraphe dix-sept nous offre un bon exemple de ce que peut provoquer l'inattention d'un copiste: Le mythe raconte ici que 'l'Homme de vie et lumière qu'il était, se changea en âme et en intellect, la vie se changeant en âme, la lumière en intellect.'10

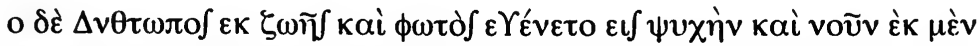
$\zeta \omega \tilde{\jmath} \int \psi v \chi \eta \dot{v} v, \varepsilon \kappa \delta \dot{\varepsilon} \phi \omega \tau \partial \int$ voũv.

et le latin nous dit: "Non autem ex vita et luce in animam, mortemque processit, vita quippe animam largita est, lux denique mortem." Il n'y a pas ici de raison évidente pour laquelle Ficin introduirait la notion de mort qui ne peut se rattacher au contexte. Si nous consultons la traduction italienne de $\mathrm{T}$. Benci à cet endroit, l'on trouve: “Ma l'huomo da la vita e da la luce, procedette in anima e in mente. Certo da la vita ricevette l'anima, e da la luce la mente". Voila la traduction fidèle d'après laquelle nous pouvons remonter à l'original. Ficin avait bien compris et avait certainement écrit "mentem". Mais cette erreur a été reprise par les éditions successives, sans même y prêter attention. Il y a plusieurs autres cas de ce genre. Pour ne citer que le premier traité du Corpus Hermeticum, nous lisons au paragraphe cinq: "ex hac luminis voce verbum factum prodiit". Il est bien évident ici que nous avons une erreur en ce qui concerne le mot $\alpha \gamma \iota f$; Benci traduit: "Si manifesto il Verbo Santo". C'est donc 'sanctum' la traduction originale de Ficin. 
Au paragraphe vingt-trois, nous lisons 'peccatum examinat', qui ne va pas avec le contexte. C'est bien entendu 'exanimat' qui est la vraie leçon. Benci traduit: 'esanima'.

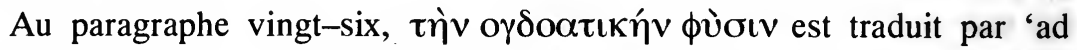
optatam naturam.' La vraie leçon est sûrement 'octavam', car Benci traduit: "salendo ritorna a la ottava natura", ce qui correspond au grec. Il est à supposer que l'étude des autres traités multipliera la découverte de ce genre d'erreurs.

Il semble que l'on puisse déterminer distinctement deux types d'erreurs de traduction assez caractéristiques de Ficin dans ce texte. D'une part, certaines de ces erreurs découlent d'un manque de compréhension; le texte est obscur en grec, mal compris de Ficin, et par là-même mal traduit. D'autre part, sa foi chrétienne l'influence également. Dans certains cas, il comprend de façon chrétienne ce qui ne l'est pas; dans d'autres, il omet tout simplement ce qui lui semble ne pas convenir à un texte chrétien.

Dès les premiers paragraphes, l'on aperçoit que Ficin tend à raccourcir les phrases, à oublier certains termes. Est ici décrite la révélation d'un maître à son disciple: souvent Ficin ne saura pas départager les répliques qui appartiennent à Hermès de celles qui doivent être attribuées à Pimandre. Il peut changer la personne du verbe, même le temps. Ainsi il traduira

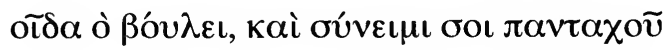

par "ac tu vide quid velis, ipse vero tibi ubique adero."11 Pour rendre le terme ođ $\delta \alpha$, il se sert de la racine opó $\omega$, et donc traduira par 'videre'12. Peu après, Pimandre recommande à Hermès de bien préparer ses questions:

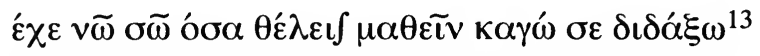

"Garde bien dans ton intellect tout ce que tu veux apprendre, et moi, je t'instruirai." Ficin traduit: "Tua me mente complectere et ego te in cunctis quae optaris erudiam". Il a transformé l'unique proposition du texte grec en deux propositions juxtaposées et surtout il a rajouté un élément de traduction, qui à notre sens, n'y était pas. Le pronom 'me' n'est pas dans le texte grec. De plus, le sens de 'complectere' peut également être "embrasser par amour". $\mathrm{Ne}$ pourrait-il s'agir ici d'une image philosophique, d'un embrassement mystique, le dieu s'unissant d'amour au fidèle, et l'initiant aux mystères?

Nous étions déjà habitués dans nos travaux précédents à rencontrer des exemples de cette liberté de Ficin par rapport à l'original. Il s'agissait alors en général d'un changement délibéré. Mais ici, nous nous posons la question: de savoir quelle était l'expérience de Ficin comme traducteur à l'époque où 
nous nous trouvons, c'est-à-dire en 1463 ? Il en est encore à ses débuts comme hélléniste. Possédant de solides bases de grec, il ne s'y est remis sérieusement que relativement tard, à la demande de son mécène, Cosme de Medicis. ${ }^{14}$ En 1463, lorsqu'il traduit Hermès, il est encore loin de la maîtrise souhaitée. Nous devrons attendre encore vingt ans pour découvrir ses traductions de Platon, pour lesquelles nous savons d'ailleurs qu'il fut aidé par certains de ses collègues. ${ }^{15}$ Dans ces derniers textes, sa traduction est certainement plus aisée; à l'inverse, pour notre part, nous croyons que la traduction du Pimandre est l'oeuvre d'un débutant, et que c'est au moins une des raisons qui lui font commettre tant d'erreurs.

Le paragraphe quatre en est truffé: il est vrai que le texte grec est difficile, obscur à la fois sur le plan du vocabulaire et sur celui du contenu. Nous lisons ici une description du chaos originel et de la séparation entre la lumière et l'obscurité. La syntaxe n'est absolument pas respectée; les expressions sont

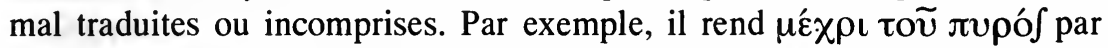
"mediam reginem inter ignem", ce qui est assez loin du grec. L'impression est ici que Ficin, ayant peine à comprendre les détails du texte, s'efforce de donner au moins un sens général au paragraphe.

Au paragraphe huit, Ficin s'engage de nouveau dans un faux sens: Pimandre explique à Hermès: "Tu as vu dans le Noûs la forme archétype, le préprincipe antérieur au commencement sans fin."16

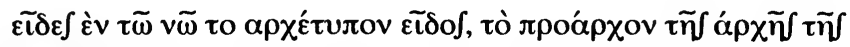

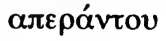

Ficin traduit: "Vidisti in mente primam speciem infinito imperio praevalentem". Tout d'abord, Ficin transforme une phrase affirmative en interrogative sans raison apparente. Ensuite, il comprend le mot $\alpha \rho \chi \eta^{\prime}$ dans son sens de 'imperium'. ${ }^{17}$ De même pour $\pi \rho o ̀ \alpha \rho \chi 0 \int:$ "praevalens", dans le sens de supériorité et non d'antériorité. Or le texte grec est ici très clair. Il y est question de la genèse du monde, de l'archétype de l'univers qui n'est pas encore créé. Le mot $\alpha \rho \chi \eta ́$ dans le sens de commencement est également le premier mot de la Bible, et certainement connu de Ficin. ${ }^{18}$ Comment a-t-il pu ne pas voir cette signification évidente? Il est difficile de l'expliquer, sinon en invoquant son inexpérience, et peut-être aussi le manque de temps; il a traduit Hermès en quelques mois seulement: rude début pour un traducteur.

Au paragraphe quinze, à la suite du mythe de la création de l'homme, la double nature humaine est établie: l'homme est mortel de par son corps et 
immortel de par son âme. Suit une phrase explicative, équilibrée de la même façon que la précédente.

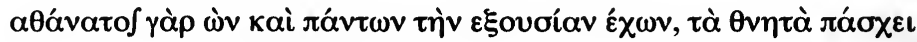

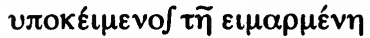

"Bien qu'il soit immortel en effet, et qu'il ait pouvoir sur toutes choses, il subit la condition des mortels, soumis, comme il l'est à la Destinée."19

Mais curieusement, Ficin ne voit pas la similarité des ces deux phrases successives. Ce n'est pas l'homme qui est soumis à la Destinée et mortel, ce sont les autres créatures vivantes: "Immortalis enim est, cunctorumque arbitrium obtinet; caetera vero viventia quae mortalia sunt, fato subjecta patiuntur." Ficin ne veut-il voir que l'immortalité de l'âme, ou bien n'a-t-il pas compris le texte? Sur le plan grammatical, sa traduction ne se tient en aucune façon. Le manuscrit qu'il possédait contenait le mot

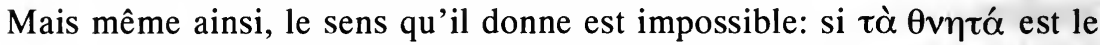
sujet, quel est l'objet? C'est d'ailleurs ainsi qu'il transcrit sa phrase latine; nous avons 'patiuntur' sans objet. De plus, il juge utile de rajouter le terme 'viventia' qui n'existe pas dans le grec. Tout ceci donnerait plutôt à penser que le texte est mal compris et ainsi, mal traduit. La phrase suivante ne change pas cette impression: elle commence par un 'non igitur' qui n'y a que faire, et ne correspond pas au grec.

Le paragraphe vingt est, nous semble-t-il, le plus déconcertant du point de vue de la traduction, non pas tant à cause du contenu plus ou moins bien rendu, mais surtout à cause de la répartition des répliques.

"Trismeg. Quot tamen delinquunt, ignorantes, inquam, ut ob eam causam immortalitate priventur? Pimand. Videris o Mercuri non satis intelligere quae audieris. Trism. Etsi nondum intelligere sim professus, intelligo tamen ac memini. Pimand. Gratular si quae dicta sunt, tenes. Trism. Responde mihi quaeso. Pimander, cur digni morte sint ii, qui in morte jacent? Pim. Quia praecessit proprio corpori tristis umbra, ex hac quidem natura humida, ex hac vero corpus in mundo sensibili constitit, ex hoc denique mors ipsa scaturiit. Num hoc tenes Mercuri?"

Ficin coupe, enlève, rajoute, et change la logique des phrases telle qu'elle

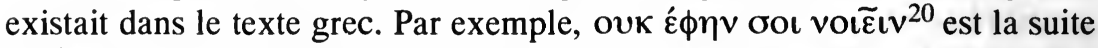

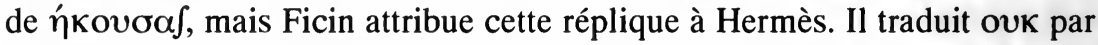
'etsi' qui introduit donc une subordonnée concessive avec une nouvelle principale. Ainsi de suite. Il continue à répartir les différentes phrases, jusqu’à 
arriver à faire poser une question à Pimandre par Hermès, alors que c'est en fait le contraire: nous assistons ici à un dialogue entre le maître et l'élève, et le premier fait répéter la leçon apprise au second. Il doit reprendre les termes

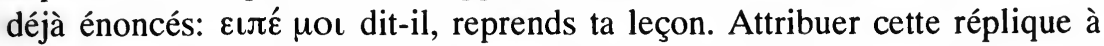
Hermès est d'autant moins logique qu'il a déjà posé cette question au début du paragraphe. D'ailleurs, dès les lignes suivantes, le maître approuve la

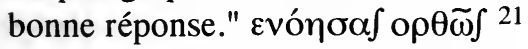

Le paragraphe six est part importante de la doctrine hermétique et donc d'un intérêt primordial pour Ficin. Le maître enseigne à son disciple: La lumière est Dieu, Noûs, et le Verbe issu de la lumière, est le fils de Dieu: "Sic inquit: cogita, quod in te videt, et audit Verbum Domini, mens autem pater Deus."

Cette dernière idée est assez répandue dans le monde héllénistique. Mais la suite est plus confuse: Par analogie, l'on peut comprendre "Ce qui en toi voit et entend, est le Verbe du Seigneur, et le Noûs est le Dieu Père."22

Dans une discussion passionnante, dont nous ne donnons pas ici les détails, C.H. Dodd ${ }^{23}$ analyse tous les éléments philosophiques de ce texte et en arrive à

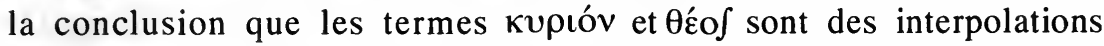
chrétiennes. Nous aurions donc ici le logos de l'homme qui est rejeton de son esprit (mens) de même que le Verbe créateur est le fils du Noûs éternel. Mais pour Ficin, il n'y avait pas de doute sur l'authenticité du texte. Avec ferveur, il apporte ici un témoignage chrétien et traduira "verbum Domini" et "Pater Deus".

Plus loin, nous voyons de nouveau Ficin pris au piège de son enthousiasme: l'Homme et la Nature, unis d'amour, créent sept hommes correspondant aux natures des sept gouverneurs (sphères du système solaire) Pimandre explique à Hermès "l'homme avait en lui la nature de l'assemblage des sept, composés je t'ai dit, de feu et de souffle." ${ }^{24}$ Le texte du manscrit de Ficin ccmporte

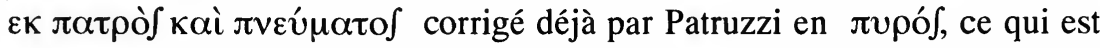
bien plus logique, la nature des gouverneurs nous étant déjà décrite précédemment au paragraphe neuf, comme étant de feu et de souffle. Mais Ficin n'a pas vraiment réfléchi au sens: pouvait-il résister à la tentation de traduire: “...nam cum septem illorum harmonia ipse jam fuisset imbutus ab eo, quem tibi paulo ante narravi, patre videlicet atque spiritu, natura ipsa non restitit."? Nous voyons encore là le signe d'un manque de réflexion.

Pour finir cette démonstration, nous nous contenterons d'indiquer deux derniers points intéressants:

Au paragraphe vingt-six, lorsque Pimandre décrit l'ascension de l'homme vers le Père, il parle longuement des Puissances qui accueillent l'âme de l'homme parmi elles et qui ensemble "entrent en Dieu". Nulle part dans le texte de Ficin, il n'est fait mention de ces Puissances $\delta v v \alpha \mu \varepsilon 1 \int$. Ficin 
supprime cette partie, probablement un peu trop païenne à son goût. Supprimer les phrases embarrassantes est donc une solution au problème. Nous retrouvons cette même attitude au paragraphe vingt-neuf: les nouveaux fidèles célèbrent Dieu et "ils furent nourris de l'eau d'ambroisie". 25 Ficin traduit: "Sapientiae sermones illorum auribus infundebam, quo factum est, ut illi ex imbrium procellis emerserint." C'est une image poétique, mais d'ambroisie, point. Il faut supposer que Ficin ne peut introduire une image si typiquement mythologique dans sa traduction. Ceci nous montre de nouveau à quel point il ne peut être un traducteur réellement indépendant de son éducation chrétienne.

Nous n'avons donné dans les précédentes pages que les exemples les plus frappants de ce traité. On pourrait aisément les multiplier, mais leur relation en deviendrait fastidieuse. Il semble que, même dans un texte relativement court, l'on puisse déjà avoir une notion de ce que nous pourrons découvrir en étudiant de façon analogue les traités suivants de Corpus Hermeticum.

Ficin n'est pas un traducteur littéral. Sur le plan de la syntaxe et de l'ordre des mots, il est loin de respecter l'original grec. Il ne tente même pas de rendre le rythme de la phrase grecque. Cela en soi ne serait pas une erreur, si ce n'était également aux dépens du sens. C'est justement ce qu'il nous faut comprendre. Ficin est un philosophe qui ne voit d'autre possibilité d'élargir ses propres connaissances et celles des autres qu'en traduisant. Sa compréhension est guidée par une foi chrétienne, qui, malgré son intelligence et son ouverture d'esprit, lui fait à l'occasion manquer à sa tâche et trahir le texte original. Parfois, comme nous l'avons vu dans le cas de citations traduites, c'est dans l'intention d'introduire son propre argument philosophique et d'y ajouter le poids d'une autorité reconnue, mais même dans le cas d'un texte suivi, il n'échappe pas à cette tendance personnelle.

Il nous semble qu'à propos du Pimandre, d'autres éléments entrent en jeu: travaillant à sa première traduction importante, il a peu de temps à sa disposition et peu d'expérience. Tout ceci se combine pour donner une traduction inexacte, mais une traduction tout de même. Vers la fin du discours, lorsque Ficin traduit l'eulogie en latin, l'on pourrait presque croire à une prière chrétienne. Son enthousiasme fut certainement ressenti par ses lecteurs: ainsi qu'il le sera plus tard pour Platon, il fut réellement le propagateur de ces textes hermétiques parmi les savants de la Renaissance. Peut-être son époque, qui vivait le renouveau mais aussi une synthèse originale des valeurs culturelles de l'antiquité, était-elle la plus apte à recevoir le message mystique, symbole d'un syncrétisme religieux, qu'apportait Hermès Trismégiste. 


\section{Notes}

1 M. Ficino, Opera Omnia, Bâle, 1576. Reproduction photographique: Turin, 1959, II, p. 1836. "Argumentum Marsilii Ficini Florentini in librum Mercurii Trismegisti.

2 I. Klutstein, Marsile Ficin et la théologie ancienne, Oracles chaldaïques-Hymnes orphiques-Hymnes de Proclus, Florence, L.S. Olschki, 1987.

3 cf. P.O. Kristeller, Supplementum Ficinianum, I, CXXIX, et également K.H. Dannenfeldt, dans Catalogus Translationum et Commentariorum, ed. P.O. Kristeller, I, pp. 138-139.

4 cf p.7.

5 R. Reitzenstein, Poimandres, Leipzig, 1904, et voir discussion sur l'histoire du texte dans Hermès Trismégiste, tome I, Paris, les Belles-lettres, éd. Nock et Festugière, 1972, pp. XI-LIII. Nous nous référons à cette édition ainsi qu'à l'excellente traduction du texte grec tout au long de cette étude.

6 F. Purnell Jr, "Hermes and the Sybil: a note on Ficino's Pimander," Renaissance Quarterly, 30 (1977), pp. 305-310.

7 voir les arguments critiques contre Ficin se basant sur une fausse tradition du texte, F. Purnell, op.cit.

8 T. Benci, il Pimandro di Mercurio Trismegisto, Firenze, 1549.

9 F. Yates, Giordano Bruno and the Hermetic tradition, Londres, 1964. ch. II, pp. 20-43. Dans son analyse, Mme Yates prend en considération les "Commentaires" qui suivent la traduction après chaque traité. Suivant ici la thèse très convaincante de P.O. Kristeller, nous considérerons que ceux-ci ne sont pas de Ficin. cf. Suppl. Fic., I, CXXX, et Studies in Renaissance Thought and Letters, Rome, 1984, pp. 221-248.

10 éd. Festugière, I. 17, 1. 20-21.

11 éd. Festugière, 1,2,1.10.

12 La même traduction se retrouve dans les Hymnes Orphiques (59) Ficin traduit oĩde par "inspicit". Mais à cet endroit, la vue et la conscience sont prises dans le même sens, dans le cadre d'un chapitre où les mots "videt" et "intuetur" reviennent souvent. cf. I. Klutstein, op. cit. p. 30.

13 éd. Festugière, $1,3,1.14$

14 A propos des connaissances de Ficin en grec, on peut avoir un aperçu du sujet dans R. Marcel, Marsile Ficin, Paris, les Belles-Lettres, 1958, pp: 243-250.

15 cf. J. Hankins: "Some remarks on the history and character of Ficino's translation of Plato" dans G.C. Garfagnini ed. Marsilio Ficino e il ritorno di Platone, Studi e Documenti, Florence, L.S. Olschki, 1986, I, pp. 288-297.

16 éd. Festugière, I,8,1.10-11.

17 Dans le lexique grec-latin, édité par R. Pintaudi, Rome, 1977, nous trouvons également pour $\alpha \rho \chi \eta ্$ les termes latins suivants: "Magistratus, Principatus, Principium" mais aucun autre sens.

18 cf. C.H. Dodd, The Bible and the Greeks, Londres, 1934, pp. 108-112.

19 éd. Festugière, I,15,1. 20-22.

20 éd. Festugière, I,20.1.20

21 éd. Festugière, I,21,1.26. 
22 éd. Festugière, I,6,1.18-20.

23 C.H. Dodd, op. cit. pp. 118-119. Plusieurs solutions différentes ont été proposées par les divers chercheurs, pour éclaircir le passage, mais celle suggérée par Dodd semble la plus logique.

24 éd. Festugière, I,16.1.8.

25 éd. Festugière, I,29,1.8-10. 